\title{
From Discovery to Finished Products: Innovative Applications of Surface Mass Spectrometry in Pharmaceutical Industry
}

\author{
Nari Talaty ${ }^{1}$, Hai Hua Gong ${ }^{2}$, Stormy Koeniger ${ }^{1}$, Andrew Vogt ${ }^{3}$, Maurice Pheil ${ }^{3}$, Peter Fruehan ${ }^{1}$, Joseph \\ Neilly ${ }^{3}$, Matthew Lopour ${ }^{2}$, Robert W. Johnson ${ }^{1}$ \\ ${ }^{1}$ Discovery Chemistry and Technologies, Research and Development, AbbVie, North Chicago, IL \\ ${ }^{2}$ Manufacturing Science and Technology, Operations, AbbVie, North Chicago, IL \\ ${ }^{3}$ NCE Analytical, Research and Development, AbbVie, North Chicago, IL
}

Surface mass spectrometry allows the direct analysis of the surface composition of materials or biological tissues, which eliminates sample processing, minimizes solvent consumption, and can increase efficiency by 5-10 times compared to standard LC-MS methods. Two mass spectrometry ionization methods, desorption electrospray ionization (DESI) and matrix assisted laser desorption ionization (MALDI) applied to surface analysis and imaging will be presented.

DESI-MS combines a novel surface sampling technique with mass spectrometric analysis. DESI is an ambient MS ionization method in which the sample is analyzed rapidly outside the vacuum in an ambient environment. In a DESI experiment a high velocity charged droplet spray is directed toward an analyte/surface of interest. These solvent droplets interact with the surface to form ions that are subsequently detected and analyzed by a mass spectrometer [1]. The advantages of this approach are the speed and ease of analysis. The implementation of DESI has enabled the molecular analysis of multiple sample types not accessible by more established mass spectrometry (MS) and other analytical techniques. DESI is employed to address product authentication, anti-counterfeit testing initiatives, polymer analysis and product quality testing. These results have been successfully correlated with attenuated total reflectance mid-infrared spectroscopy, atomic force microscopy and scanning electron microscopy data. MALDI mass spectrometry imaging (MSI) provides the distribution of both targeted and untargeted set of molecules from a surface such as a tissue section. Understanding the distribution of pharmaceutical compounds throughout the tissue section can provide insightful information regarding mechanism of action, toxicity, lack of efficacy and optimized dosing regimens. Tumor drug levels are common endpoints in developing anti-cancer drugs; however, in traditional LC-MS methods the spatial distribution throughout the tumor is lost. MSI has been applied to determine distribution and penetration of molecules in tumor as well as in brain and skin tissue sections. Recent developments in quantitative methods for MSI will also be presented [2].

In the pharmaceutical industry, DESI has demonstrated broad utility. A Prosolia Omnispray DESI source with 1D automated motion control capability fitted onto an AB Sciex QTrap 5500 mass spectrometer was applied to the analysis of polymers and tablets. Quantitative (standard MRM) and fingerprinting (Ion-trap profiling) assays were developed. DESI was applied to characterize the surface chemistry composition of a molded polymer component in order to predict the performance of the part during use. DESI was also developed as a minimally invasive and sensitive anti-counterfeiting tool and used for product authentication. Spectral fingerprints were obtained from the surface of tablets to detect API and excipients and compared with suspected counterfeits (Fig 1). Coatings of tablets were analyzed by the DESI technology as an investigative tool for a manufacturing process. DESI was used to identify small spots of extraneous matter found on surfaces of tablets or polymers such as impurities, ink spots and dyes. These results were compared and combined with findings from other microscopic methods. 
Mass spectrometry imaging (MSI) was applied to drug discovery programs in oncology (Fig 2), neuroscience and dermatology. Case studies highlighting the importance of spatial distribution of pharmaceutical and endogenous compounds in tumor, brain and skin sections will be presented. MSI data was combined with immunohistochemistry (IHC) and histological staining, and an indirect quantitation method for MSI was developed. Quantitation of MSI images was accomplished by calibration of MSI data to LC-MS/MS concentrations. MSI was performed on an oMALDI-QSTAR Elite. In conclusion several applications of surface mass spectrometry, using DESI and MALDI, spanning from early drug discovery to finished product testing were implemented.

\section{References:}

[1] H. Chen, N. Talaty, Z. Takats and R.G. Cooks, Anal Chem., 77 (2005), p. 691527.

[2] S.L. Koeniger et al., Rapid Commun Mass Spectrom., 25 (2011), p.503-10

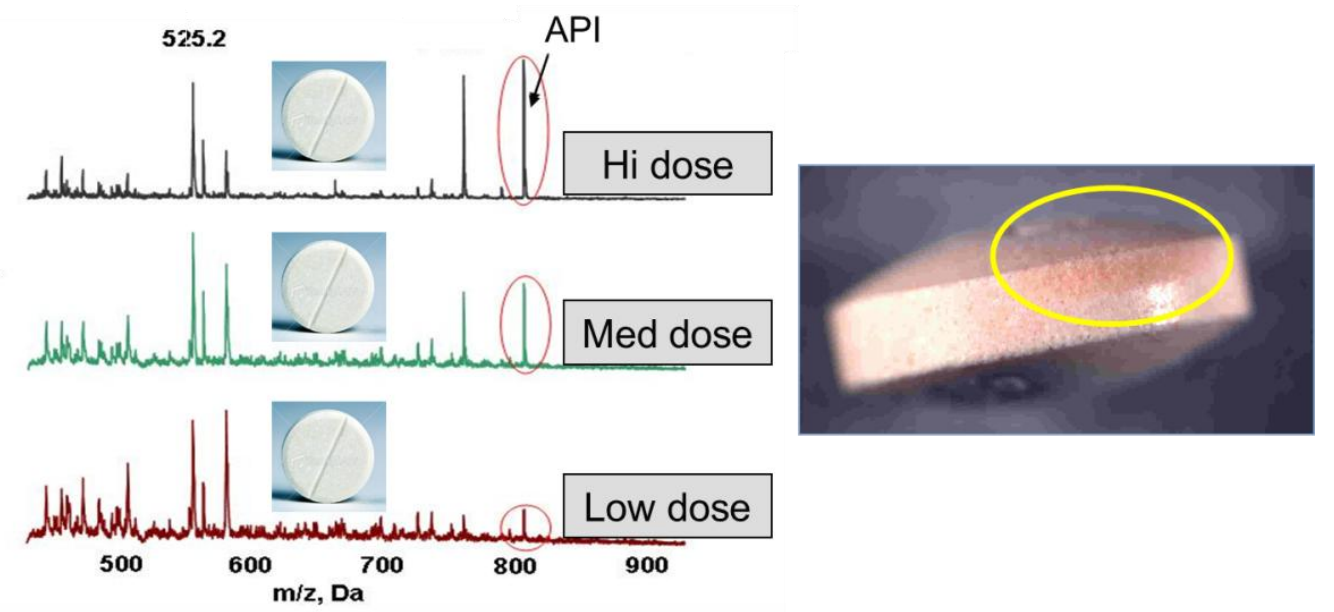

Figure 1. Mass spectra of three tablets showing fingerprint comprising of excipient and API peaks, enabling authentication. Picture of tablet post analysis: DESI is minimally invasive, sample is preserved.
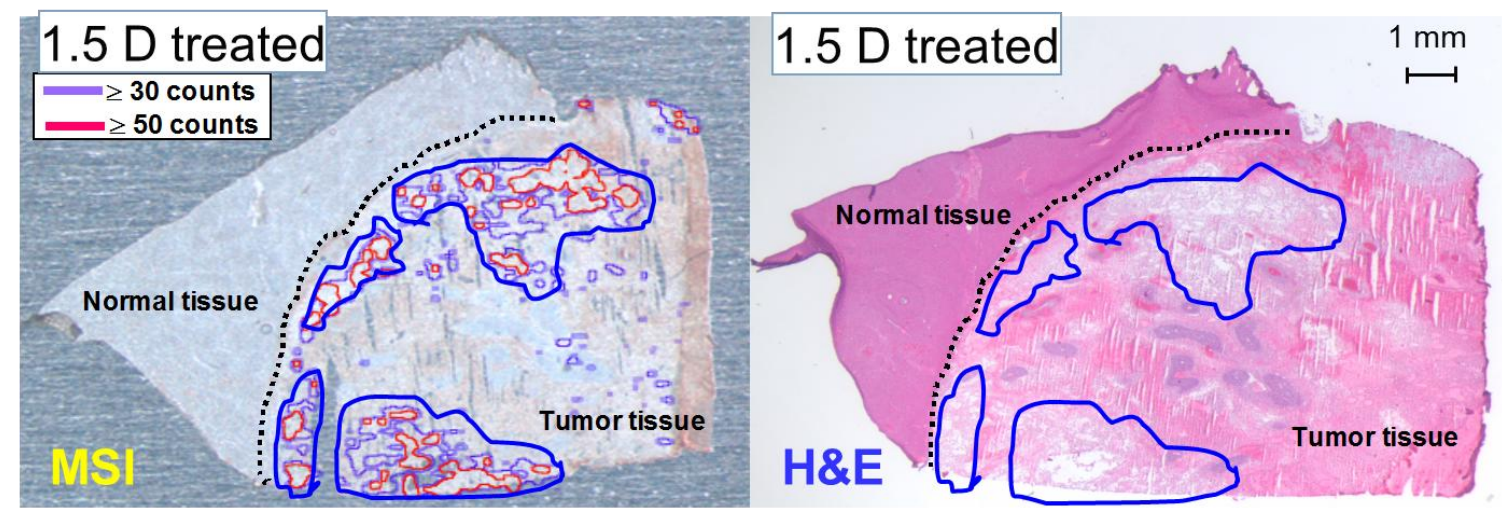

Figure 2. Left: Mass spectrometry image of a tumor tissue showing the distribution of compound. Right: H\&E stained image of an adjacent tissue section used to identify the tumor region and boundary.

\section{Disclosures:}

All authors are employees of AbbVie. The design, study conduct, and financial support for this research were provided by AbbVie. AbbVie participated in the interpretation of data, review, and approval of the publication. 